\title{
An Intelligent LMS+F Algorithm
}

\author{
Dimitrios I. Pazaitis and A.G. Constantinides \\ Signal Processing Section, Dept. Electrical and Electronic Engineering \\ Imperial College, Exhibition Road, London SW7 2BT, UK \\ e-mail : \{d.pazaitis, a.constantinides\}@ic.ac.uk
}

\begin{abstract}
A new techique for combining the LMS and LMF cost sunctions is proposed in this contribution. The resulting stochastic gradient adaptive algorithm uses a time varying mixing parameter to optimise a combination of the above cost functions, taking into consideration the noise statistics. Furthermore, the behaviour of the proposed algorithm is analysed and convergence conditions are established. Simulation results verify the ability of the algorithm to adapt itself to the noise characteristics, illustrate its enhanced perfor. mance and support very well the theoretic analysis. The continuous adaptation of the mixing parameter adds fiexibility and enables rapid response of the algorithm to non stationarities.
\end{abstract}

\section{Introduction}

Stochastic gradient adaptive algorithms are quite popular and have been used in a wide variety of applications including array processing, system identification, channel equalization, echo and other interference cancellations, mainly due to their inherent simplicity. LMS is the mostly known such algorithm and its performance has been thoroughly investigated in the literature (e.g $[4,5,7,12]$ ). Its most attractive feature is its amenability to simple implementation, while its main drawback is the degradation of its performance due to eigenvalue spread.

The LMS algorithm belongs to a more general family, which attempts to optimize (minimize) the following cost function [12]

$$
J=E\left\{e^{2 K}(n)\right\} \quad K=1,2, \ldots
$$

and is obtained by setting $K$ equal to 1, i.e., $J=E\left\{e^{2}(n)\right\}$. For $K=2$, we obtain the second member of the family, i.e., $J=E\left\{e^{4}(n)\right\}$, known as the Least Mean Fourth (LMF) algorithm. Walach and Widrow compared the two algorithms above and found out that under certain conditions the LMF outperforms LMS [12]. Furthermore, it is obvious that when far from the optimum (i.e., $e(n)>1$ ) the LMF algorithm exhibits faster convergence.

The $L M S+F$ algorithm [8] was a first approach towards the combination of the advantages of the two algorithms. More precisely, $L M S+F$ aimed at exploiting the faster initial convergence of the LMF algorithm, while retaining the desir-

This work was supported by a scholarship from the State Scholarship Foundation (I.K.Y) of the Hellenic Republic. able LMS characteristic of low misadjustment and immunity over the different distributions of noise, when around the optimum.

A mixed criterion algorithm of this type, obtained though from a different perspective, was also presented in [3]. The proposed algorithm was derived in an attempt to minimise the variance of the square error subject to a constraint on the mean square error. The constrained minimisation method resulted in a weight update formula with a fixed preselected mixing parameter. However, no information was provided about the optimal value of the mixing parameter or the constraint. The proposed combination appeared also in [1], where a constant mixing parameter $\lambda$ was used. In this contribution, the $\lambda$ selection criterion of [8] is modified, so that the evolution of the $\lambda$ sequence takes into account the noise characteristics, enhancing thus the algorithm's performance. Moreover, the adaptive nature of the $\lambda_{n}$ sequence adds flexibility to our algorithm.

\section{The LMS+F Adaptive Algorithm}

The following type of cost function is proposed

$$
J=\left(1-\lambda_{n}\right) E\left\{e^{2}(n)\right\}+\lambda_{n} E\left\{e^{4}(n)\right\},
$$

where $\lambda_{n}$ is a time varying scalar sequence and $\alpha$ is a scaling factor, selected according to the following rule

$$
\lambda_{n+1}=\left\{\begin{array}{ll}
\lambda_{n}+\alpha & , \text { if } E\left\{e_{n}^{4}\right\} \geq 1 \\
\lambda_{n}-\alpha \operatorname{sgn}\left\{C_{e}^{4}(n)\right\} & , \text { otherwise }
\end{array},\right.
$$

where sgn is the sign (signum) function, $C_{e}^{4}(n)=E\left\{e_{n}^{4}\right\}-$ $3 E\left\{e_{n}^{2}\right\}$ is the kurtosis (fourth order cumulant) of the assumed zero mean error signal and $\alpha$ is a scaling factor lying in the interval $[0,1]$. By choosing $\alpha=1.0$ no "transient" behaviour is exhibited and the algorithm just alternates between LMS and LMF. To preserve the unimodal characteristic of the mixed cost function, as both of the consisting functions are characterised by convexity, $\lambda_{n}$ in (2) is confined to the closed interval $[0,1]$.

As it can be seen from the first part of (3), when far from the optimum weight vector, i.e., $e(n)>1, \lambda_{n}$ increases, increasing therefore the convergence speed, but as the weight vector $H_{n}$ approaches its optimum value $\left(\boldsymbol{H}^{\text {opt }}\right), \lambda_{n}$ adjusts itself according to the noise distribution, enhancing therefore the algorithms's performance. 'The first part of the above formula implicitly assumes that the noise variance is lower than unity. If this is not the case, it can be 
either ignored or automatic gain control or normalisation with respect to the input power could be applied to enable the use of the above update equation, which significantly improves the initial convergence of the $L M S+F$ algorithm in non stationary environments [8]. Applying the steepest descent gradient search, and using the instantaneous value of the gradient instead of the mean, the filter coefficient vector update equation for the cost function (2) becomes

$$
\boldsymbol{H}_{\boldsymbol{n}+\mathbf{1}}=\boldsymbol{H}_{\boldsymbol{n}}+2 \boldsymbol{\mu}\left(\left(1-\lambda_{n}\right) e(n)+2 \lambda_{n} e^{3}(n)\right) \boldsymbol{X}_{\boldsymbol{n}},
$$

where

$$
e(n) \triangleq w_{n}-X_{n}^{T} V_{n}
$$

and the weight error vector is given by

$$
V_{n}=H_{n}-H_{n}^{o p t}
$$

We can now proceed to analyse the proposed algorithm.

\section{Convergence analysis of the LMS+F algorithm}

To facilitate our analysis we introduce the commonly used assumption that the various input vectors come from mutually independent zero mean gaussian distributed sequences $[2,4,5,12]$. Although this is untrue in many applications, since consecutive input vectors share $N-1$ entries, it is widely accepted to capture the first order behaviour and is extensively used in the literature to simplify the analysis producing at the same time reliable results [7]. In our case, the assumption can be relaxed, in that we only require that the input sequence is uncorrelated with the filter weights. Other than that, no restriction applies to the nature of the input autocorrelation matrix $\boldsymbol{R}$.

We also approximate the conditional expectation terms of the form $E\left\{e_{n}^{2} \mid V_{n}\right\}$ with the unconditional mean square estimation error. For slow adaptation conditions, the weight error vector oscilates around the mean value justifying partially the above assumption. Furthermore, according to the central limit theorem, as the filter length increases, the distribution of the error signal gets closer to the gaussian one. This assumption is not new and has led to analytic results which agreed well with the simulated behaviour of nonlinear algorithms $[2,6]$.

Finally, the estimation error $e_{n}$ is assumed to follow a gaussian distribution. The above assumption is justified, when the weight vector $H_{n}$ varries much slower than the input vector $X_{n}$; a condition corresponding to the slow adaptation case. This assumption has produced reliable results and was used succesfully in $[6,11]$. The approximate validity of the above assumptions will be confirmed by the simulation results.

By substracting $H^{o p t}$ from both sides of equation (4) using (6) and (5) results in

$$
\begin{aligned}
V_{n+1} & =V_{n}+\mu\left(\left(1-\lambda_{n}\right)\left(w_{n}-X_{n}^{\boldsymbol{T}} V_{n}\right)\right) \\
& +\mu\left(2 \lambda_{n}\left(w_{n}-X_{n}^{\boldsymbol{T}} V_{n}\right)^{3}\right) \boldsymbol{X}_{n} .
\end{aligned}
$$

We wish now to develop a recursive equation for the time evolution of the correlation matrix of the weight error vector
$V_{n}$. Using $K_{n}$ to denote this correlation matrix at time instant $n$, we have, by definition,

$$
K_{n} \triangleq E\left\{V_{n} V_{n}^{T}\right\}
$$

Substituting (7) in (8) we obtain

$$
\begin{aligned}
& E\left\{\boldsymbol{V}_{\boldsymbol{n}+\mathbf{1}} \boldsymbol{V}_{n+1}^{\boldsymbol{T}}\right\}=E\left\{\boldsymbol{V}_{\boldsymbol{n}} \boldsymbol{V}_{\boldsymbol{n}}^{\boldsymbol{T}}\right\} \\
& \quad+E\left\{\mu\left(\left(1-\lambda_{n}\right) e_{n}+2 \lambda_{n} e_{n}^{3}\right)\left[\boldsymbol{V}_{n} \boldsymbol{X}_{\boldsymbol{n}}^{\boldsymbol{T}}+\boldsymbol{X}_{n} \boldsymbol{V}_{n}^{\boldsymbol{T}}\right]\right\} \\
& \quad+E\left\{\mu^{2}\left(\left(1-\lambda_{n}\right) e_{n}+2 \lambda_{n} e_{n}^{3}\right)^{2} \boldsymbol{X}_{n} \boldsymbol{X}_{n}^{\boldsymbol{T}}\right\}
\end{aligned}
$$

To obtain the individual terms on the right hand of the above equation we take conditional expectations (with respect to $V_{n}$ ) and then average over all $\boldsymbol{V}_{\boldsymbol{n}}$. We thus obtain

$$
E\left\{e_{n}\left(\boldsymbol{V}_{n} \boldsymbol{X}_{n}^{\boldsymbol{T}}+\boldsymbol{X}_{n} \boldsymbol{V}_{n}^{T}\right)\right\}=-\left(\boldsymbol{K}_{n} \boldsymbol{R}+\boldsymbol{R} \boldsymbol{K}_{n}\right) .
$$

Adopting a similar approach and applying Price's [10] theorem on the right hand terms of (9), we obtain [9]

$$
\begin{aligned}
E\left\{e_{n}^{3}\left(\boldsymbol{V}_{n} \boldsymbol{X}_{n}^{\boldsymbol{T}}+\boldsymbol{X}_{n} \boldsymbol{V}_{n}^{\boldsymbol{T}}\right)\right\} & =-3 \sigma_{e_{n}}^{2}\left(\boldsymbol{K}_{n} \boldsymbol{R}+\boldsymbol{R} \boldsymbol{K}_{n}\right), \\
E\left\{e_{n}^{2} \boldsymbol{X}_{n} \boldsymbol{X}_{n}^{\boldsymbol{T}}\right\} & =\sigma_{e_{n}}^{2} \boldsymbol{R}+2 \boldsymbol{R} \boldsymbol{K}_{n} \boldsymbol{R} \\
E\left\{e_{n}^{4} \boldsymbol{X}_{n} \boldsymbol{X}_{n}^{\boldsymbol{T}}\right\} & =3 \sigma_{e_{n}}^{4} \boldsymbol{R}+12 \sigma_{e_{n}}^{2} \boldsymbol{R} \boldsymbol{K}_{n} \boldsymbol{R},
\end{aligned}
$$

and

$$
E\left\{e_{n}^{6} \boldsymbol{X}_{n} \boldsymbol{X}_{n}^{\boldsymbol{T}}\right\}=15 \sigma_{e_{n}}^{8} \boldsymbol{R}+90 \sigma_{e_{n}}^{4} \boldsymbol{R} \boldsymbol{K}_{n} \boldsymbol{R} .
$$

Substituting (10), (11), (12), (13) and (14) in (9) we obtain the following equation for the error correlation matrix

$$
\begin{aligned}
& \boldsymbol{K}_{n+1}=\boldsymbol{K}_{n}-\mu\left(\left(1-\lambda_{n}\right)+6 \lambda_{n} \sigma_{e_{n}}^{2}\right)\left(\boldsymbol{K}_{n} \boldsymbol{R}+\boldsymbol{R} \boldsymbol{K}_{n}\right) \\
& +\mu^{2}\left(\left(1-\lambda_{n}\right)^{2} \sigma_{e_{n}}^{2}+12 \lambda_{n}\left(1-\lambda_{n}\right) \sigma_{e_{n}}^{4}+60 \lambda_{n}^{2} \sigma_{e_{n}}^{6}\right) \boldsymbol{R} \\
& +\mu^{2}\left(2\left(1-\lambda_{n}\right)^{2}+48 \lambda\left(1-\lambda_{n}\right)+360 \lambda^{2} \sigma_{e_{n}}^{4}\right) \boldsymbol{R} \boldsymbol{K}_{\boldsymbol{n}} \boldsymbol{R},
\end{aligned}
$$

Due to the form of the above equation, i.e., non linear, an exact convergence condition is difficult to find. To fascilitate our analysis we introduce the concept of the distance $T_{n}[11]$

$$
T_{n}=\operatorname{tr}\left[\Lambda K_{n}^{\prime}\right]=\operatorname{tr}\left[R K_{n}\right]=E\left\{\left(V_{n}^{\boldsymbol{T}} X_{n}\right)^{2}\right\} .
$$

Adopting the approach in [4] we develop the following sufficient and necessary condition on the step size parameter that ensures mean square convergence

$$
0<\mu_{\max }<\frac{2\left(\left(1-\lambda_{n}\right)+6 \lambda_{n}\left(\sigma_{w}^{2}+T_{n}\right)\right)}{\left(I_{1}+I_{2}\right)\left(N+2 I_{3}\right) \gamma_{\max }},
$$

where

$$
\begin{aligned}
& I_{1}=\left(1-\lambda_{n}\right)^{2}+12 \lambda_{n}\left(1-\lambda_{n}\right)\left(2 \sigma_{w}^{2}+T_{n}\right) \\
& I_{2}=60 \lambda_{n}^{2}\left(3 \sigma_{w}^{4}+3 \sigma_{w}^{2} T_{n}+T_{n}^{2}\right) \\
& I_{3}=\left(1-\lambda_{n}\right)^{2}+24 \lambda\left(1-\lambda_{n}\right)+180 \lambda^{2}\left(\sigma_{w}^{2}+T_{n}\right)^{2}
\end{aligned}
$$

and $\gamma_{\max }$ stands for the maximum eigenvalue of the input correlation matrix $\boldsymbol{R}$. 


\section{Simulation results}

In this final section we present and analyse the results obtained from simulations. The algorithm is applied to a system identification problem, where the system to be identified is considered non stationary. The optimum filter coefficients assume the following initial values $\boldsymbol{H}_{0}^{\text {opt }}=[0.2,0.4$, $0.6,0.8,1.0,1.0,0.8,0.6,0.4,0.2]$ and, after that, experiences random disturbances. The null vector $(\mathbf{0})$ is chosen as the initial vector - starting point - $H_{0}$, and all the results are obtained by averaging over an ensemble of 100 runs. The parameter $\lambda$ is initialised to 1 , i.e. we start with the LMF algorithm. The input is assumed gaussian distributed and both noise and input sequences are assumed to be zero mean i.i.d with input and noise variance equal to unity and 0.1 respectively. The System mismatch $\left(E\left\{V_{n}^{\boldsymbol{T}} V_{n}\right\}\right)$ is selected as a performance measure. The mean square and the mean fourth value of the error are estimated using the following formula

$$
E\left\{e_{n}^{k}\right\}=\beta E\left\{e_{n-1}^{k}\right\}+(1-\beta) e_{n}^{k},
$$

where $k=2,4$ and the constant $\beta$ is a memory controlling factor. The larger the value it assumes the "stronger" the memory of the system. Alternatively, a finite length moving window could be used.

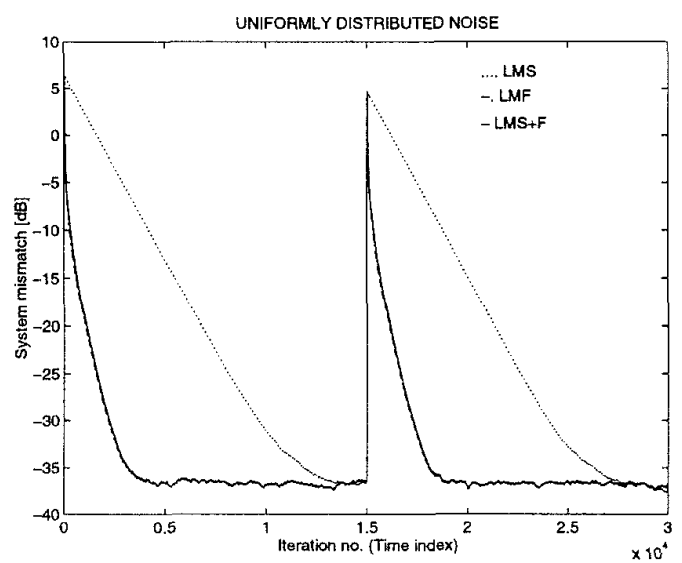

Figure 1: Performance comparison between the LMS (dotted line), the $L M F$ (dash dot line) and the $L M S+F$ algorithm (solid line) under uniformly distributed noise conditions

Figures 1,2 and 3 depict the performance behaviour of all the algorithms, namely LMS, LMF and $L M S+F$, under various noise conditions. The parameters of the above algorithms were chosen so as either to match the steady state error (misadjustment) or the convergence rate, depending on the insight in the performance of the mixed algorithm they provide. The chosen values are as follows: $\mu_{L M S}=4.5 \cdot 10^{-4}, \mu_{L M F}=\mu_{L M S+F}=1.8 \cdot 10^{-3}$ for the uniformly distributed noise case (figure 1) and $\mu_{L M S}=$ $\mu_{L M F}=\mu_{L M S+F}=1.8 \cdot 10^{-3}$ for the gaussian and laplacian distributed noise cases in figures 2 and 3 respectively. The random disturbances are assumed to follow a uniform dis-

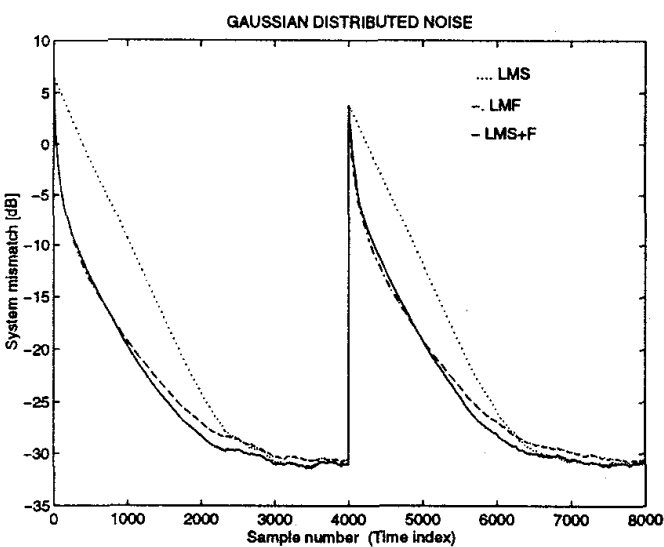

Figure 2: Performance comparison between the LMS (dotted line), the $L M F$ (dash dot line) and the $L M S+F$ algorithm (solid line) under gaussian distributed noise conditions

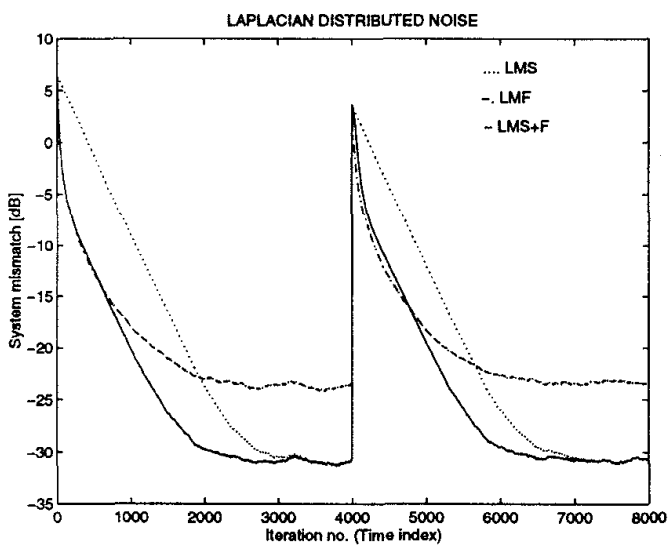

Figure 3: Performance comparison between the LMS (dotted line), the $L M F$ (dash-dot line) and the $L M S+F$ algorithm (solid line) under laplacian distributed noise conditions

tribution with variance $\sigma_{A}^{2}=0.3$ in figure 1 and $\sigma_{A}^{2}=0.25$ in figures 2 and 3.

In figure 1 we see that the $L M S+F$ algorithm behaves as the LMF providing fast convergence and low steady state error. To achieve the same steady state error with the LMS algorithm, we had to significantly decrease the convergence factor $\mu$.

In figures 2 and 3 we observe that the $L M S+F$ adopts the initial high tracking speed of the LMF algorithm but, as it approaches the optimum, it gradually changes to the LMS algorithm (laplacian noise case) or combines both criteria (gaussian noise case) to achieve a lower steady state error.

The adaptability of the $L M S+F$ algorithm to time varying noise distributions is shown in figure 4 , where its performance is also compared with that of LMS and LMF. As in the previous figures, the $L M S+F$ exploits the fast initial convergence of the $\mathrm{LMF}$ algorithm and after that adopts the 


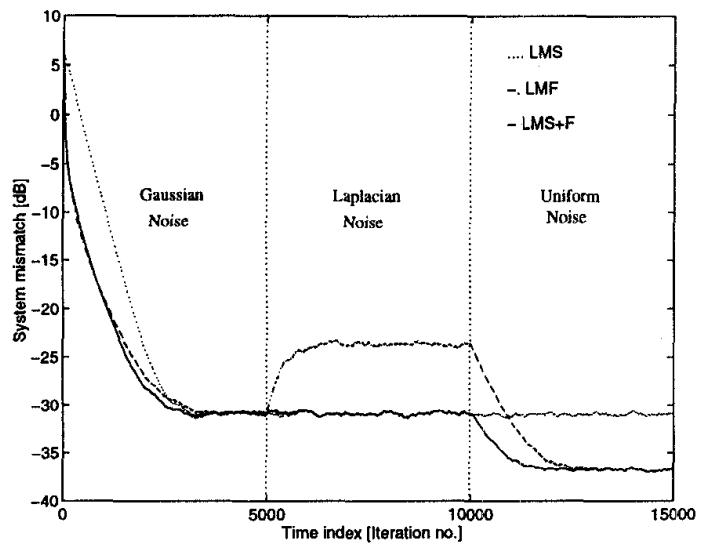

Figure 4: Performance curves of the algorithms under time varying noise conditions (LMS: dotted, LMF: dash dot, $L M S+F:$ solid)

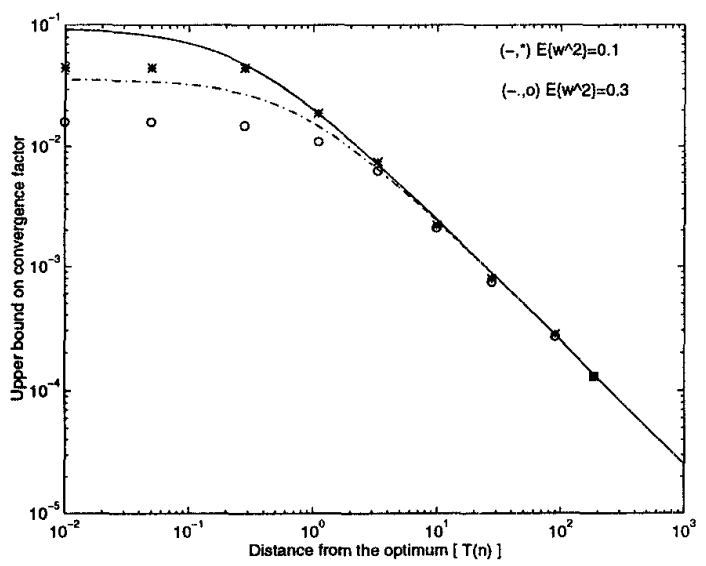

Figure 5: Comparison between the theoretically obtained upper bound on $\mu$ (solid $\left(\sigma_{w}^{2}=0.1\right)$ and dashed $\left(\sigma_{w}^{2}=0.3\right)$ lines) and and the ones obtained from simulations (stars $\left(\sigma_{w}^{2}=0.1\right)$ and circles $\left.\left(\sigma_{w}^{2}=0.3\right)\right)(\lambda=\delta=1 / 2)$.

performance criterion that exhibits the lower steady state error.

Next, we focus our attention on the theoretically obtained upper allowable value for the convergence factor, which is a function of the parameter $\lambda$. When $\lambda$ is allowed to take any value in the closed interval $[0,1]$ the convergence condition is dictated by that of LMF (i.e., $\lambda=1$ ), since it poses more stringent convergence bounds. However, if, for any reason, the $\lambda_{n}$ sequence is constrained to $[0, \delta]$, where $\delta<1$, then the necessary and sufficient condition is obtained from equation (17) by setting $\lambda$ equal to $\delta$. This latter case is depicted in figure 5 , where a comparison between the expected (Eq.17) and observed upper bounds is presented for two values of noise variance and $\delta$ equal to $1 / 2$. It is easily observed that the simulation results are in good agreement with the theoretical ones, especially when far from the optimum. The observed deviation from the the- oretic curves near the optimum is justified by noting that the theoretical results are obtained using averages, whereas in simulations, we deal with the instantaneous values of the stochastic variables. It can be also observed that, when far from the optimum, the condition on the step size is dominated by the distance $T_{n}$ and is almost independent of the noise variance.

\section{Conclusions}

In this contribution, a new technique for mixing the LMS and LMF cost functions was presented. It differs from what was previously suggested in that it is time varying and takes into account the noise distribution. The proposed algorithm was analysed and conditions regarding the behaviour and stability were established. Simulation results verify the improved performance of the proposed algorithm and support well the theoretic results.

\section{References}

[1] J.A. Chambers, O. Tanrikulu, and A.G. Constantinides. Least mean mixed-norm adaptive filtering. Electronics Letters, 30(19):1574-1575, September 1994.

[2] S. C. Douglas and T. H.-Y. Meng. Stochastic Gradient Adaptation Under General Error Criteria. IEEE Trans. Signal Processing, 42(6):1335-1351, June 1994.

[3] J. D. Gibson and S. D. Gray. MSVE Adaptive Filtering Subject to a Constraint on MSE. IEEE Trans. Circuits and Systems, 35(5):603-608, May 1988.

[4] Simon Haykin. Adaptive Filter Theory. Englewood Cliffs : Prentice-Hall, 2nd edition, 1991.

[5] L. L. Horowitz and K.D. Senne. Performance advantage of complex LMS for controlling narrow-band adaptive arrays. IEEE Trans. Acoustics, Speech and Signal Processing, ASSP-29(3):722-735, June 1981.

[6] V. J. Mathews. Performance Analysis of Adaptive Filters Equipped with the Dual Sign Algorithm. IEEE Trans. Signal Processing, 39(1):85-91, January 1991.

[7] J.E. Mazo. On the Independence Theory of Equalizer Convergence. The Bell System Technical Journal, 58(5):963-993, 1979.

[8] D. 1. Pazaitis and A. G. Constantinides. LMS+F Algorithm. Electronics Letters, 31(17):1423-1424, August 1995.

[9] D.I. Pazaitis. Adaptive Signal Processing Techniques for Improved Convergence and Noise Robustness. MPhil/PhD transfer report, Imperial College, March 1996.

[10] R. Price. A Useful Theorem for Nonlinear Devices Having Gaussian Inputs. IRE Trans. Information Theory, IT. 4:69-72, June 1958.

[11] O. Tanrikulu and A.G. Constantinides. Mean square convergence analysis and variable step-size of the LMF adaptive algorithm. Submitted in Trans. Circuits and Systems, 1995

[12] E. Walach and B. Widrow. The Least Mean Fourth (LMF) Adaptive Algorithm and its Family. IEEE Transactions on Information Theory, 30(2), March 1984 . 